\title{
Yes-associated protein (YAP) promotes progression of glioma and poor prognosis of human
}

\section{Fei Yan}

Liaocheng Brain Hospital

Lele Du

Liaocheng Brain Hospital

Jiatao LV

Liaocheng Brain Hospital

Haitao Zhang

Liaocheng Brain Hospital

Jianxin Zhu

Liaocheng Brain Hospital

Fuhua Yu ( $\sim 519494381 @ q q . c o m)$

Liaocheng Brain Hospital

\section{Research Article}

Keywords: Glioma, YAP, Migration, hippo signaling pathway, prognosis

Posted Date: August 13th, 2021

DOl: https://doi.org/10.21203/rs.3.rs-808708/v1

License: (c) (i) This work is licensed under a Creative Commons Attribution 4.0 International License.

Read Full License 


\title{
Yes-associated protein (YAP) promotes progression of glioma and poor prognosis of human
}

\author{
Fei Yan ${ }^{1}$,Lele Du ${ }^{1}$,Jiatao Lv $^{1}$, Haitao Zhang ${ }^{1}$,Jianxin Zhu ${ }^{1}$, Fuhua Yu ${ }^{1 *}$ \\ feiYan:yanfei5459@163.com; \\ leleDu:564360387@qq.com; \\ JiataoLv:Ivjiatao2021@163.com; \\ HaitaoZhang:zht0000082021@16.com;
}

Jianxin Zhu:zjxIcrm@163.com;

Fuhua Yu:519494381@qq.com.

${ }^{1}$ Department of Neurosurgery, Liaocheng Brain Hospital, Liaocheng252000, Shandong Province, China

1 Background: Yes-associated protein(YAP) plays an important role in signal transduction and gene transcription regulation in

2 normal cells, with elevated and over-expressed YAP levels observed in various malignant tumors. The aim of this study was to investigate the expression of YAP in malignant glioma, and to study the possible relationship of YAP expression with the

4 occurrence and development of malignant glioma.

5 Methods: Immunohistochemical staining was used to assess the expression of YAP and phosphor-YAP in malignant glioma

6 tissue and normal brain tissue, and their protein and mRNA levels were evaluated through Western blotting and reverse

7 transcription-polymerase chain reaction (RT-PCR), respectively. Normal brain tissue obtained from the functional lesion of

8 the epilepsy patients. After transfection of YAPsiRNA oligonucleotides or pcDNA3.1-hYAP plasmid, their effects on glioma

9 cells were investigated using western blot, cell proliferation, cycle, apoptosis and invasion, respectively. We conducted the 
co-Immunoprecipitation to verify the combination of YAP and PPAR $y$, explore the mechanism of action.

11 Results: YAP-positive expression was found in 9 cases of normal brain and 60 cases of glioma. A significantly higher expression of YAP in glioma tissue as compared with normal brain tissue at both protein and mRNA levels, and YAP proteins mainly expressed and located in the nucleus and only a small percentage in the cytoplasm of glioma tissue. Phosphor-YAP protein expression showed high staining of the cytoplasm, but no staining of the nuclear. While, with the enhancement of the malignant degree, the cytoplasm YAP(p-YAP) expression is lower gradually than normal brain tissues. Further study in glioma cell lines in which YAP was either overexpressed or depleted confirmed that YAP markedly promoted the cell proliferation, cycle, invasion and inhibited the cell apoptosis. Moreover, YAP in company with PPARy regulates the cell proliferatin and effects the gliomagenesis.

Conclusion: These results indicate that YAP plays an important role in glioma and might be a useful therapeutic target of glioma.

Keywords: Glioma, YAP, Migration, hippo signaling pathway, prognosis

\section{Introduction}

Malignant gliomas, such as glioblastoma multiforme (GBM), are the most common and aggressive malignant primary brain tumors. Despite the advances in surgery, radiation therapy, and chemotherapy, the prognosis of patients with GBM has not been improved significantly over the past 20 years $^{[1]}$. It is imperative to have a detailed and comprehensive understanding of the molecular pathogenesis of the gliomas for developing novel strategies in treatment. The Hippo pathway is an important regulator of cell growth, proliferation, and apoptosis. It was first discovered by genetic mosaic based screens in Drosophila melanogaster ${ }^{[2,3]}$; however, there is an increasing body of evidence demonstrating that the Hippo pathway also limits organ size in mammalian systems ${ }^{[4]}$ by inhibiting cell proliferation and promoting apoptosis. Components of this pathway are highly conserved in mammals, and include Mst1/2; WW45; Lats1/2; Mob1; YAP; TAZ; NF; 
FRMD6; and Fat4 ${ }^{[2]}$. Mst1/2 and Last1/2 are kinases, and WW45 and Mob1 act as adaptors/activators in mammals, together these make up the core kinase cassette of the Hippo pathway. The Yki homologs:Yes-associated protein (YAP) and its paralog, transcriptional co-activator with PDZ-binding motif (TAZ), are the principle targets of the core Hippo kinase cascade, and are considered to be the nuclear effectors of Hippo pathway ${ }^{[5]}$.

Previous studies have reported that aberrant alteration of the key components of the Hippo pathway leads to uncontrolled cell growth, and is associated with cancer development ${ }^{[6,7]}$. This implies that the Hippo pathway plays a critical role in suppressing tumor growth ${ }^{[8]}$. Aberrant activation of YAP has been associated with poor prognosis in multiple of human cancers ${ }^{[9,10]}$, and may act as an tumor suppressor gene in breast cancer ${ }^{[11]}$. As such, YAP has been proposed as a candidate oncogene. Furthermore, overexpression of YAP was found to enhance liver size and eventually lead to tumor development in conditional transgenic mice models ${ }^{[12,13]}$, indicating that YAP may be involved in tumor development and progression as a carcinogenic factor. This study aimed to examine YAP expression, determined the function of YAP in glioma and explored the possible mechanism of YAP.

\section{Material and Methods}

\section{Subjects and Sample collection}

A total of 60 cases of glioma and 9 cases of normal brain tissues were retrieved from the archives of the Liaocheng People's hospital Neurosurgery department from 2014 to 2017. The histological diagnosis and grade of differentiation were evaluated by two independent pathologists, S.Z.Y. and B.C.S., according to the 2004 World Health Organization classification. As controls, the normal brain tissues were obtained from patients who underwent internal decompressive surgery after physical injuries to the brain. Clinicopathological information about the patients was obtained from patient records, and is summarized in Table 1. All of the enrolled patients underwent curative surgical resection without having prior chemotherapy or radiation therapy. The study was approved by the hospital ethical committee. 
Formalin-fixed, paraffin-embedded tissue microarrays were bought from the Shanxi chaoying tissue microarray core facility.

54 We graded the YAP expression according to the distribution, intensity, and percentage of positive cells. Absence of reactivity was graded as negative. For the cytoplasmic distribution, weak cytoplasmic reactivity was considered as low expression regardless of extent. Strong cytoplasmic reactivity with less than $50 \%$ positive cells was graded as low expression, otherwise it was graded as high expression. For the nuclear distribution, nuclear expression in less than $10 \%$ of cells was graded as low expression and nuclear expression in more than $10 \%$ cells was graded as high expression. Samples with low or high YAP staining were classified as YAP positive ${ }^{[6]}$. Immunohistochemistry for YAP was performed as follows: Slides were deparaffinized in xylene and rehydrated in a graded alcohol series. Antigen retrieval was achieved by microwaving in $10 \mathrm{mmol} / \mathrm{L}$ of sodium citrate buffer at $\mathrm{pH} 6 \mathrm{for} 10 \mathrm{~min}$. Peroxidase was blocked with $3 \%$ hydrogen peroxide in methanol, and nonspecific protein binding was blocked with $5 \%$ goat serum. The primary antibody for YAP(Cell Signaling Technology, USA; catalog\#4912) was diluted (1:50) with phosphate buffered saline (PBS) and applied overnight in a humidity chamber at $4{ }^{\circ} \mathrm{C}$, meanwhile, using $20 \mu$ of PBS as a negative control.Slides were then covered with $20 \mu$ l of horseradish peroxidase (HRP)-conjugated goat anti-rabbit secondary antibody (Beijing Zhongshan Goldenabridge Biotechnology Co.Ltd.,China;1:100 dilution) for 30 minutes.Detection was performed with diaminobenzidine(DAB) chromogenic reagent(Beijing Zhongshan Goldenabridge Biotechnology Co.Ltd.).Sections were counterstained with hematoxylin, dehydrated and mounted.Five visual fields $(\times 200)$ from each slide were analyzed with Leica Qwin V3 system to obtain the average gray value for each slide.Scoring for the YAP staining was performed as previously described.

\section{Transfection}

The plasmid of pcDNA3.1-hYAP was obtained from GenScript (nanjing)Co.,Ltd.pcDNA3.1-hYAP or control pcDNA3.1 plasmid was transfected into U87 and 118 glioma cells using X-tremeGENE HP DNA Transfection Reagent(Roche,Germany) according to the manufacturer's instructions. In the next experiments, we also transduced the PPARY plasmid to the U87 
and 118 glioma cell which had transfented the pcDNA3.1-hYAP plasmid. Then we observed the glioma cell growth by MTT 76 assay.

77 The YAPsiRNA were obtained from Genepharma Co,Ltd(Shanghai,China): (5'-CUG CCA CCA AGC UAG AUA ATT--3'),targeting the YAP gene; and the negative control duplex,(5'--UUA UCU AGC UUG GUG GCA GTT--3').Oligonucleotides $(20 \mathrm{pmol} / \mu \mathrm{l})$ were transfected into SNB19 and LN229 cells at 70\% confluence using X-tremeGENE siRNA Transfection reagent according to the manufacture's instruction (Roche,Germany).

\section{$81 \quad$ qRT-PCR Analysis}

Total RNA from tissue sample and glioma cells was isolated using Trizol reagent (Invitrogen,USA) for YAPmRNA analysis.For analysis of YAPmRNA expression, real-time RT-PCR analysis was carried out.Amplification reaction was performed with MJ-real-time PCR(Bio-Rad,Hercules,CA,USA) using Hairpin-itTM mRNAs qPCR Quantitation Kit according to the manual.Relative gene expression was calculated using the $2^{-{ }^{\triangle}}{ }_{\mathrm{CT}}$ method and analyzed initially using Opticon Monitor Analysis Software V2.02 software(MJ Research,Waltham,MA,USA), normalized to the espression of GAPDH.All qRT-PCRs assays were performed in triplicates, and the data are presented as means \pm standard errors of the means(SEM).

\section{Western Blot Analysis}

Total protein was respectively extracted from sample tissues and glioma cells using sodium dodecylsulfate(SDS) lysis buffer containing phenylmethyl sulfonylfluoride(PMSF).Total protein(30 $\mu \mathrm{g})$ was seperated by $10 \%$ SDS-PAGE and then electrophoretically transferred onto a polyvinylidene fluoride(PVDF) membranes(Millipore, Bedford, MA).The membrane was blocked with $5 \%$ dry milk and incubated overnight at $4{ }^{\circ} \mathrm{C}$ with rabbit polyclonal antibodies against YAP(Cell Signaling Technology, USA; catalog\#4912,1:500 dilution;).The $\beta$-actin protein served as an internal control. After washing, the membrane was incubated with a horseradish peroxidase-conjugated secondary antibody(Beijing Zhongshan Goldenabridge Biotechnology Co.Ltd., China) at $37^{\circ} \mathrm{C}$ for $2 \mathrm{~h}$.Protein bands were visualized with the enchanced chemiluminescence (Pierce) 
and detected using Biolmaging System(UVP, Upland, CA,USA).

\section{$98 \quad$ Cell proliferation Assay}

99 Glioma cells were seeded into 96 -well plates at 4000 cells per well. After transfection as described previously, on each day of consecutive 6 days, $20 \mu \mathrm{MTT}(5 \mathrm{mg} / \mathrm{mL})$ was added into each well and the cells were incubated at $37^{\circ} \mathrm{C}$ for additional 4 hours, and the supernatant was discarded. The reaction was then terminated by lysing the cells with $200 \mu$ l of DMSO. Optical density was measured at wavelength of $490 \mathrm{~nm}$ and expressed as percentage of control. The data are presented as the mean $\pm S E M$, which were derived from triplicate samples of at least three independent experiments.

\section{Flow Cytometric Analysis}

For analysis of cell cycle kinetics by flow cytometry, transfected and control cells in the log phase of growth were harvested, washed with PBS, fixed with $90 \%$ ethanol overnight at $4{ }^{\circ} \mathrm{C}$, and then incubated with $\mathrm{RNase}$ at $37^{\circ} \mathrm{C}$ for 30 minutes.Nuclei of cells were stained with propidium iodide for additional 30 minutes.A total of 10000 nuclei were examined in a FACS Calibur flow cytometer (Becton-Dickinson, USA) and DNA histograms were analyzed by Modifit software.

\section{Annexin V staining}

Parental and transfected cells were harvested at 48 hours post-transfection.The apoptosis was analyzed by using AnnexinV-FITC Apoptosis Detection Kit(BD Biosciences, USA) according to the manufacture's instruction. AnnexinV-FITC and propidium iodide double staining cells detected by FACS Calibur were evaluated as the apoptotic cells. The data obtained were analyzed with CellQuest software.

\section{Transwell Assay}

For analyzing the invasive activity of GBM cells, the upper surface of the transwell filters was coated with matrigel.Cells in the logarithmic growth phase were harvested. Cells $\left(1 \times 10^{5}\right)$ in $200 \mu$ l of serum-free DMEM were added into the upper chamber. A total of $600 \mu$ l of conditioned medium derived from tumor cell culture was used as a source of chemoattractant 
and placed in the lower chamber. After 24 -hour incubation at $37^{\circ} \mathrm{C}$, the filters were gently taken out, the medium was removed from the upper chamber. The non-invaded cells on the upper surface of the inserted filter were scraped off with a cotton swab. The cells that had migrated into the lower surface of the inserted filter were fixed with methanol.The number of cells invading through the matrigel was counted using three randomly selected visual fields under inverted microscope.

\section{Co-Immunoprecipitation}

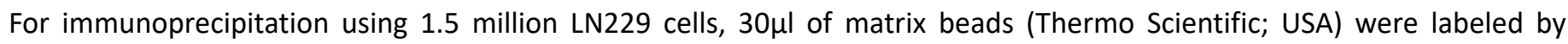
resuspension in $200 \mu \mathrm{l}$ of calcium-free PBS containing $10 \mu \mathrm{g}$ of antibody, incubated for $1 \mathrm{~h}$ at room temperature with end-over-end mixing using a rotator, washed twice with calcium-free PBS, and resuspended in $100 \mu$ l of lysis buffer (50 mM Tris $\mathrm{HCl}, \mathrm{pH} 7.4,1 \%$ Triton $\mathrm{X}-100$ and protease inhibitors). The cells were resuspended for $15 \mathrm{~min}$ in $110 \mu \mathrm{l}$ of lysis buffer. After full-speed centrifugation of the cell lysate $\left(10 \mathrm{~min}\right.$ at $\left.4^{\circ} \mathrm{C}\right), 10 \mu \mathrm{l}$ of supernatant was collected for protein expression analysis and the remaining $100 \mu \mathrm{l}$ was subjected to immunoprecipitation by incubation with the antibody-labeled beads overnight at $4^{\circ} \mathrm{C}$ using a rotator. Centrifuge the beads for 30 seconds at $5,000-8,200 \times \mathrm{g}$. Remove the supernatants with a narrow-end pipette tip. Wash the beads three times with $500 \mu$ l of ice-cold TBS. Immunoprecipitated proteins were released by incubation of the beads in SDS-PAGE loading buffer $(50 \mathrm{mM}$ Tris $\mathrm{HCl}, \mathrm{pH} 7.4,10 \%$ glycerol, $2 \%$ SDS, $0.05 \%$ bromophenol blue, $100 \mathrm{mM} \beta$-mercaptoethanol) for $3 \mathrm{~min}$ at $95^{\circ} \mathrm{C}$. Non-immunoprecipitated proteins previously collected were used as a control for protein expression level.

\section{Statistical Analysis}

The statistical package SPSS 18.0 (SPSS, Windows) was used for all analyses as described previously. All values are expressed as mean \pm SD. The $\chi 2$-test was used to determine the correlation between YAP expression and clinicopathologic factors. All P-values were based on the two-sideds tatistical analysis and a P-value of $<0.05$ was considered to indicate statistical significance. 


\section{Results}

\section{Expression of YAP in human glioma and poor prognosis}

We next evaluated YAP expression and cellular location in 60 tumors of various World Health Organization(WHO) grades. Normal brain tissues $(9 / 9,100 \%)$ showed no staining of nucleus. In contrast, very high proportion of WHO grade astrocytoma (7/11,63.6\%),WHO grade II astrocytoma (8/11,72.7\%),WHO grade III glioblastoma (21/22,95.5\%),and WHO grade IVglioblastoma multiforme (GBM) (16/16,100\%) showed at least some nuclear reactivity(Table 2).Within the group of astrocytoma, high-grade tumors, including glioblastoma and glioblastoma multiforme (GBM), showed greater high level staining than normal brain tissues. Moreover, YAP expression contributes to the poor prognosis of glioma. (Figure 1) Cytoplasmic YAP(phosphor-YAP) was detected in 60 tumors of various WHO grades in line with the YAP staining. Total normal brain tissues $(9 / 9,100 \%)$ showed high staining of the cytoplasm, but no staining of the nuclear. While, with the enhancement of the malignant degree, the cytoplasm YAP(p-YAP) expression is lower gradually than normal brain tissues.

\section{(Figure 2A)}

The YAP protein was mainly accumulated in the nucleus with a lesser cytoplasmic presence (Figure 2B).We also verified by qRT-PCR and Western blot which were identical with the results above (Figure 3 ).

\section{Selection of the specific glioma cell lines}

YAP activity was analyzed in ten human glioma cell lines by assessing levels of YAP protein relative to phosphor-S127-YAP. Both the level of YAP and ratio of YAP to phosphor-S127-YAP varied greatly between cell lines (Figure 4A). The results were in line with the qRT-PCR(Table 3) of YAP expression(Figure 4B).For further study, we chose cell lines with low baseline YAP activity (U87 and 118) and the cell line that showed the highest baseline YAP activity(SNB19 and LN229), based on the fact that it had less Hippo pathway-dependent inhibitory phosphorylation at S127（Figure4）. 
To determine whether YAP plays a role in glioma cell proliferation, MTT assays was performed. The mRNA level and protein level were assessed 48 hours later of transfection. Relative expression level of YAP was analyzed by real-time PCR in a panel of glioma cell lines. The SNB19 and LN229 cell lines had higher YAP levels while the U87 and 118 cell lines had lower levels.

We knocked down YAP expression in SNB19 and LN229 cells using YAP-specific siRNA. Efficient depletion of YAP expression was confirmed by western blot and qRT-PCR analysis(Fig.5). YAP depletion in SNB19 and LN229 cells reduced the proliferation rate (Figure $7 A-B)$.

We also overexpressed YAP in U87 and 118 cells. Transfection of pcDNA3.1-hYAP plasmid significantly increased YAP mRNA and protein levels compared with control and empty vector group (Figure 6).Overexpression of YAP in U87 and 118 cells led to significant increase in the U87 and 118 cell growth rate (Figure7C-D).

\section{YAP increases the invasion of glioma cells.}

To address the impact of YAP on glioma cell invasion, we performed Matrigel invasion assay. YAP-depleted LN229 and SNB19 cells showed a weaker invasion ability $(P<0.05)$ compared with control and NC group. Overexpression of YAP in U87 and 118 cells stimulated cell invasion $(P<0.05)$ when compared with control and empty vector group. (Figure 8 )

\section{YAP Accelerates the Cycle of glioma cells in the G0/G1 Phase}

We performed the Flow Cytometric Analysis to determine whether YAP accelerates the cycle of glioma cells. To analyze whether decreased viability effect of YAPsiRNA on SNB19 and LN229 was a result of cell-cycle arrest. Treatment with YAPsiRNA resulted in a decrease in the population of cells that were in S phase.A representative experiment was shown in SNB19 cells that S phase cell population accounted for $22.49 \pm 0.79 \%$ and $22.69 \pm 0.54 \%$ in control and negative control groups, respectively, in contrast to $9.82 \pm 0.36 \%$ in YAPsiRNA treated cells. Similarly, the percentage of S phase cells decreased from $22.61 \pm 1.09 \%$ of control and $24.19 \pm 0.49 \%$ of negative control groups to $7.76 \pm 0.23 \%$ of YAPsiRNA group in LN229 cells. Compared with control and negative control groups cells, the YAPsiRNA treated cells substantially and consistently increased the G0/G1 cell population from $50.80 \pm 0.67 \%$ of control group and $53.82 \pm 0.59 \%$ of negative 
control group to $66.02 \pm 0.73 \%$ in YAPsiRNA treated SNB19 cells.LN229 cells exposed to YAPsiRNA also arrested in the G0/G1 phase $(69.42 \pm 0.83 \%)$ as compared to the control and negative control group cells( $51.55 \pm 0.74 \%$ and $51.59 \pm$ $0.58 \%$,respectively). (Figure 9A-B)

Overexpressed YAP in U87 and 118 cells with the transfection of pcDNA3.1-hYAP plasmid significantly accelerates the cell cycle $(P<0.05)$ when compared with control group and control empty vector. In U87 and 118 cells, the results shown that $S$ phase cell population accounted for $14.49 \pm 1.59 \%$ and $15.61 \pm 1.09 \%$ in control groups and $15.78 \pm 1.16 \%$ and $14.35 \pm$ $1.55 \%$ in empty vector group respectively, in contrast to $32.47 \pm 1.67 \%$ and $33.5 \pm 2.33 \%$ in pcDNA3.1-hYAP plasmid treated cells. Compared with control groups cells, the pcDNA3.1-hYAP plasmid treated cells substantially and consistently decreased the G0/G1 cell population from $74.29 \pm 1.8 \%$ and $71.72 \pm 4.07 \%$ of control group and $71.37 \pm 2.38 \%$ and $69.54 \pm$ 3.14\%of empty vector group to $51 \pm 1.1 \%$ and $52.9 \pm 2.5 \%$ in pcDNA3.1-hYAP plasmid treated SNB19 and LN229 cells, respectively ( Figure 9C-D), indicating that YAP functions as a positive regulator of the cell cycle from G0/G1-to-S transition.

\section{YAP Inhibits Cell Apoptosis}

The effect of decreased YAPmRNA on apoptosis was analyzed by conducting AnnexinV and PI double staining. Untreated cells served as a negative control. Percentages of apoptotic cells are shown in the graph. Compared with the apoptotic cells in control group (3.56 $\pm 0.33 \%$ and $2.63 \pm 0.24 \%)$ and negative control group $(2.44 \pm 0.42 \%$ and $3.68 \pm 0.46 \%)$ in SNB19 and LN229 cells, respectively, the downregulation of YAPmRNA resulted in a significant $(P<0.05)$ increase of apoptotic cells in SNB19 and LN229 cells (18.99 $\pm 0.66 \%$ and $18.8 \pm 1.03 \%$,respectively). (Figure 10 Left)

We then investigated the difference in apoptosis ratio between the overexpression YAP groups and the control and empty vector group to confirm our retrospective cohort study results. Flow cytometry analysis showed that the apoptosis ratio was decreased in U87 and 118 cells transfected with pcDNA3.1-hYAP plasmid from 9.22\%and $10.0 \%$ to $2.80 \%$ and $2.57 \%$ in cells of control groups and $9.31 \%$ and $9.08 \%$ in empty vector group, respectively. (Figure 10 Right)

Collectively, these results indicated that YAP expression played a role in promoting cell proliferation and suppressing cell 
apoptosis.

Moreover, we detected also the expression of Ki-67,MMP-9,CyclinD1,BCL-2 by Western blotting (Figure 11). The results were in line with those above.

\section{Co-Immunoprecipitation Shows the Combination of YAP and PPARY}

Co-Immunoprecipitation experiments were performed on total protein from the LN229 glioma cells showed that YAP interacted with PPARy, suggesting a function at the level of protein ( Figure 12). To determine whether PPARy plays a role in glioma cell proliferation, MTT assays was performed. When we transfected the PPARy plasmid, the proliferation ability can decrease vividly ( Figure 13).

\section{Discussion}

Glioblastoma is the most common subtype of primary brain tumor in adults. These tumors are very aggressive with a highly invasive capacity and often infiltrate critical neurological areas within the brain ${ }^{[14]}$. Glioblastoma cells are notoriously resistant to apoptosis, a characteristic that contributes toward the failure of most standard clinical treatments ${ }^{[15]}$. Current research in this area is driven by the need to discover new agents that will be effective and have few side effects.

Overexpression of YAP has been implicated in tumor progression in various human cancers ${ }^{[16]}$. However, the expression of YAP and its correlation with clinicopathologic factors have not been defined in glioma. In this study, we demonstrated that overexpression of YAP in glioma tissues was significantly correlated with grades malignancy of glioma. Furthermore, YAP overexpression was associated with poor prognosis of glioma patients.

In previous studies, YAP expression and nuclear localization was found to be elevated in hepatocellular carcinoma, prostate cancer, colon cancer, and breast cancer ${ }^{[6,10,16]}$. A study of 28 cases of lung adenocarcinoma subjected to tissue microarray showed that YAP was expressed in $54 \%$ of $\operatorname{cases}^{[6]}$. Clinical study in 177 hepatocellular carcinoma patients showed that YAP could serve as an independent predictor for hepatocellular carcinoma-specific disease-free survival and overall survival[ ${ }^{[16]}$. Consistent with these findings, the present report showed that the level of YAP was increased in glioma 
tissues, which was mainly accumulated in the nucleus with a lesser cytoplasmic presence. Importantly, YAP positive expression was associated with reduced overall survival in glioma patients, but did not serve as an independent predictor for patient survival. Taken together, these results indicate that YAP is an oncogenic protein overexpressed in glioma and is associated with glioma progression.

YAP is acknowledged as a candidate oncogene, and became the focus of research, after it was identified in human chromosome 11q22 amplicon ${ }^{[17]}$. To address the impact of YAP on proliferation in glioma, we overexpressed and silenced YAP in glioma cell lines. We found that overexpression of YAP resulted in a marked increase of cell growth rate, while depletion of YAP greatly suppressed cancer cell proliferation. Moreover, consistent with previous studies ${ }^{[10]}$, we found that YAP was able to overcome cell contact inhibition. The YAP-overexpressed U87 and 118 cells kept proliferating and resulted in a higher saturation density than control cells.

In this study, we showed that YAP overexpression significantly promoted glioma cell invasion, accelerated the cycle of glioma cells in the G0/G1 phase and inhibited the cell apoptosis. Nevertheless, YAPsiRNA knockdown blocked cell invasion, reduced cell proliferation, arrested the cycle of glioma cells in the G0/G1 phase and promoted the cell apoptosis. These results implied YAP may play a oncogene role in gliomagenesis.

Peroxisome proliferator-activated receptors (PPARs) are ligand-activated transcription factors that were first discovered 20 years ago ${ }^{[18]}$. There are three isoforms of PPAR; PPAR- $\alpha$, PPAR- $\beta / \delta$ (also known as PPAR- $\beta$ or PPAR- $\delta$ ), and PPAR- $\gamma^{[19]}$. These have been found in all the mammalian species that have been examined to date ${ }^{[20,21]}$. PPAR- $\gamma$ is highly expressed in cancer cells, and treatment with PPAR- $\gamma$ ligands can induce cell differentiation and apoptosis ${ }^{[22,23]}$. Although it remains unclear whether PPARs are oncogenes or tumor suppressors, research has also been focused on this receptor because of its involvement in various metabolic disorders that are known to be associated with cancer risk ${ }^{[24,25]}$.To further $^{2}$ explore the mechanisms by which YAP promoted glioma cells, we performed the Co-Immunoprecipitation. We observe that YAP protein can deposit PPARy mutually by western blot. Then, we transduced the PPARy plasmid into the U87 and 118 
glioma cells which had transfected the pcDNA3.1-hYAP plasmid, the cell proliferation increased significantly in contrast to control group, suggesting PPARy maybe acted as tumor suppressor gene in gliomagenesis. However,the concrete machanisms is still unknown.

However, YAP was also reported to be a tumor suppressor as its gene locus was deleted in some breast cancers with a correlated loss of YAP protein expression ${ }^{[11]}$. Yap/Taz transcriptional activity is essential for vascular regression via Ctgf expression and actin polymerization ${ }^{[26]}$.In addition, some studies have identified YAP as a transcriptional coactivator

promoting anti-tumoral effects on the basis of its interaction with p53 family members, p73, and p53-binding protein- ${ }^{[27]}$.These results present a rather intriguing question: is YAP1 an anti- or pro-tumorigenic protein? One possible explanation is that pathways are not fixed but can dynamically change depending on the context ${ }^{[28]}$. YAP contributes to glioma cell migration and invasion by regulating $\mathrm{N}$-cadherin and Twist, as well as cytoskeletal reorganization ${ }^{[29]}$.Thus, it is possible that YAP function changes depending on the upstream input, and the variations of binding partners' repertoire between cells.

In conclusion, this study identifies YAP as an oncoprotein overexpressed in glioma which is important for the regulation of malignant cell growth, invasion and apoptosis. Moreover, YAP expression contributes to the poor prognosis of glioma, making it a candidate target protein for future cancer therapeutics. Additional work is needed to elucidate the molecular functions of YAP in glioma. 


\section{References:}

1. Louis, D.N., Molecular pathology of malignant gliomas. Annu Rev Pathol, 2006. 1: p. 97-117.

2. Pan, D., Hippo signaling in organ size control. Genes Dev, 2007. 21(8): p. 886-97.

3. Edgar, B.A., From cell structure to transcription: Hippo forges a new path. Cell, 2006. 124(2): p. 267-73.

4. Lai, Z.C., et al., Control of cell proliferation and apoptosis by mob as tumor suppressor, mats. Cell, 2005. 120(5): p. 675-85.

5. Dong, J., et al., Elucidation of a universal size-control mechanism in Drosophila and mammals. Cell, 2007. 130(6): p. 1120-33.

6. Steinhardt, A.A., et al., Expression of Yes-associated protein in common solid tumors. Hum Pathol, 2008. 39(11): p. 1582-9.

7. Hong, W. and K.L. Guan, The YAP and TAZ transcription co-activators: key downstream effectors of the mammalian Hippo pathway. Semin Cell Dev Biol, 2012. 23(7): p. 785-93.

8. Olson, M.F., Modeling human cancer: report on the Eighth Beatson International Cancer Conference. Cancer Res, 2005. 65(24): p. 11247-50.

9. Zender, L., et al., Identification and validation of oncogenes in liver cancer using an integrative oncogenomic approach. Cell, 2006. 125(7): p. 1253-67.

10. Zhao, B., et al., Inactivation of YAP oncoprotein by the Hippo pathway is involved in cell contact inhibition and tissue growth control. Genes Dev, 2007. 21(21): p. 2747-61.

11. Yuan, M., et al., Yes-associated protein (YAP) functions as a tumor suppressor in breast. Cell Death Differ, 2008. 15(11): p. $1752-9$.

12. Zhang, N., et al., The Merlin/NF2 tumor suppressor functions through the YAP oncoprotein to regulate tissue homeostasis in mammals. Dev Cell, 2010. 19(1): p. 27-38.

13. Camargo, F.D., et al., YAP1 increases organ size and expands undifferentiated progenitor cells. Curr Biol, 2007. 17(23): p. 2054-60.

14. Castro, M.G., et al., Current and future strategies for the treatment of malignant brain tumors. Pharmacol Ther, 2003. 98(1): p. 71-108.

15. Ng, H.K. and P.Y. Lam, The molecular genetics of central nervous system tumors. Pathology, 1998. 30(2): p. 196-202.

16. Xu, M.Z., et al., Yes-associated protein is an independent prognostic marker in hepatocellular carcinoma. Cancer, 2009. 115(19): p. 4576-85.

17. Overholtzer, M., et al., Transforming properties of YAP, a candidate oncogene on the chromosome 11q22 amplicon. Proc Natl Acad Sci U S A, 2006. 103(33): p. 12405-10.

18. Koeffler, H.P., Peroxisome proliferator-activated receptor gamma and cancers. Clin Cancer Res, 2003. 9(1): p. 1-9.

19. Bishop-Bailey, D., PPARs and angiogenesis. Biochem Soc Trans, 2011. 39(6): p. 1601-5. https://pubmed.ncbi.nlm.nih.gov/22103494/ PMID: 22103494

20. Peters, J.M., Y.M. Shah and F.J. Gonzalez, The role of peroxisome proliferator-activated receptors in carcinogenesis and chemoprevention. Nat Rev Cancer, 2012. 12(3): p. 181-95.

21. Wang, T., et al., Peroxisome proliferator-activated receptor gamma in malignant diseases. Crit Rev Oncol Hematol, 2006. 58(1): p. 1-14.

22. Mannelli, M., et al., Role of the PPAR-gamma system in normal and tumoral pituitary corticotropic cells and adrenal cells. Neuroendocrinology, 2010. 92 Suppl 1: p. 23-7.

23. Wan, Z., et al., Peroxisome proliferator-activated receptor gamma agonist pioglitazone inhibits beta-catenin-mediated glioma cell growth and invasion. Mol Cell Biochem, 2011. 349(1-2): p. 1-10.

24. Oyekan, A., PPARs and their effects on the cardiovascular system. Clin Exp Hypertens, 2011. 33(5): p. 287-93.

25. Chen, Y.C., et al., Peroxisome proliferator-activated receptor gamma (PPAR-gamma) and neurodegenerative disorders. Mol 
Neurobiol, 2012. 46(1): p. 114-24.

26. Nagasawa -Masuda, A. and K. Terai, Yap/Taz transcriptional activity is essential for vascular regression via Ctgf expression and actin polymerization. PLoS One, 2017. 12(4): p. e0174633.

27. Matallanas, D., et al., RASSF1A elicits apoptosis through an MST2 pathway directing proapoptotic transcription by the p73 tumor suppressor protein. Mol Cell, 2007. 27(6): p. 962-75.

28. Santos, S.D., P.J. Verveer and P.I. Bastiaens, Growth factor-induced MAPK network topology shapes Erk response determining PC-12 cell fate. Nat Cell Biol, 2007. 9(3): p. 324-30.

29. Zhang Y., et al., YAP Promotes Migration and Invasion of Human Glioma Cells. Journal of Molecular Neuroscience, 2018. 64(2): p. 262-272.

Table 1 YAP Immunohistochemical Scoring System

\begin{tabular}{l|l}
\hline Score & Grading \\
\hline Complete absence of reactivity & Negtive \\
Weak cytoplasmic reactivity(regardless of extent) & Low \\
Strong cytoplasmic reactivity in less than $\mathbf{5 0 \%}$ of cells & Low \\
Strong cytoplasmic reactivity in $>\mathbf{5 0} \%$ of cells & High \\
Nuclear expression in sporadic cells $(<\mathbf{1 0} \%$ of cells) & Low \\
Nuclear expression in $>\mathbf{1 0} \%$ of cells & High \\
\hline
\end{tabular}

Table 2: Association of YAP expression in Glioma with clinical and pathological factors (chi-square test, $\mathrm{n}(\%)$ )

\begin{tabular}{|c|c|c|c|c|}
\hline & Cases & YAP positive & YAP negative & P values \\
\hline Age(years) & & & & 0.479 \\
\hline$\leqslant 60$ & 47 & 42 & 5 & \\
\hline$>60$ & 13 & 10 & 3 & \\
\hline Gender & & & & 0.898 \\
\hline Female & 25 & 22 & 3 & \\
\hline Male & 35 & 30 & 5 & \\
\hline WHO grade & & & & $0.046^{*}$ \\
\hline I & $11(18.3)$ & 7 & 4 & \\
\hline $\mathrm{II} \sim \mathrm{IV}$ & $49(81.7)$ & 45 & 4 & \\
\hline
\end{tabular}

*Statistically significant, $\mathrm{P}<0.05$. 


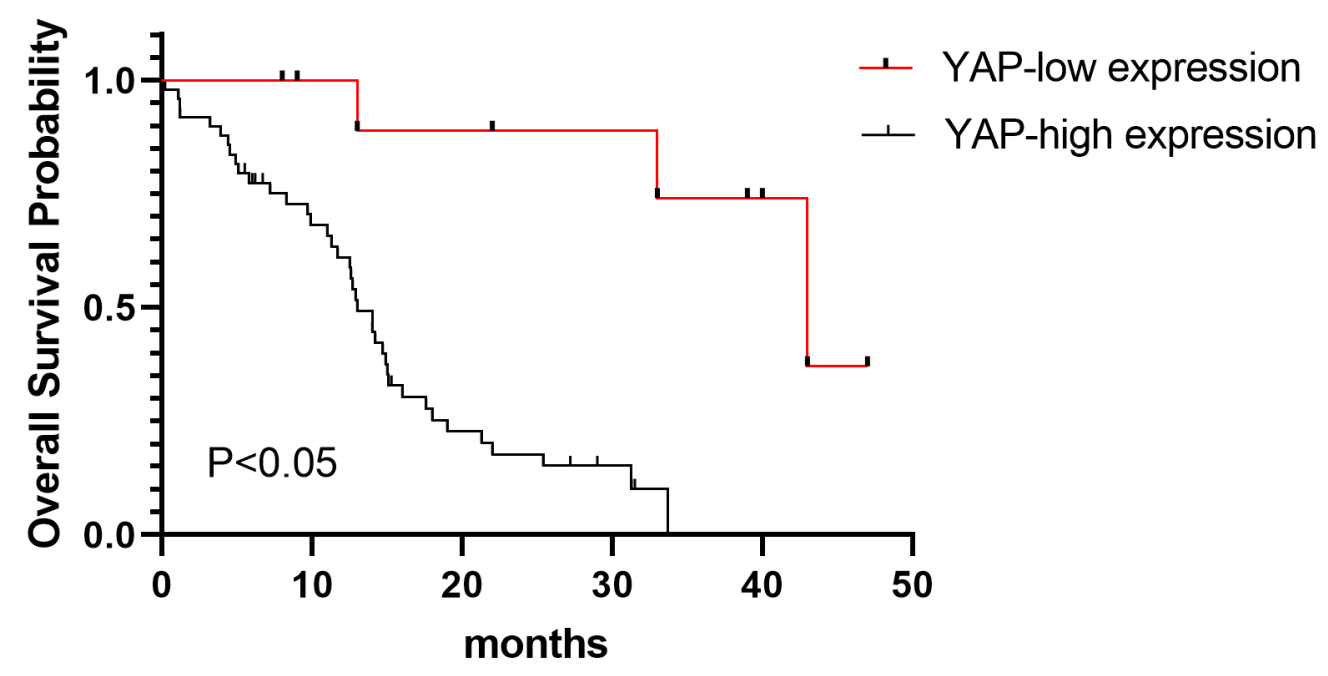

Figure1: Prognostic significance of core components of Hippo/YAP pathway. YAPexpression patterns with overall survival time were presented by Kaplan-Meier plotter based on the database integrated from Table2.
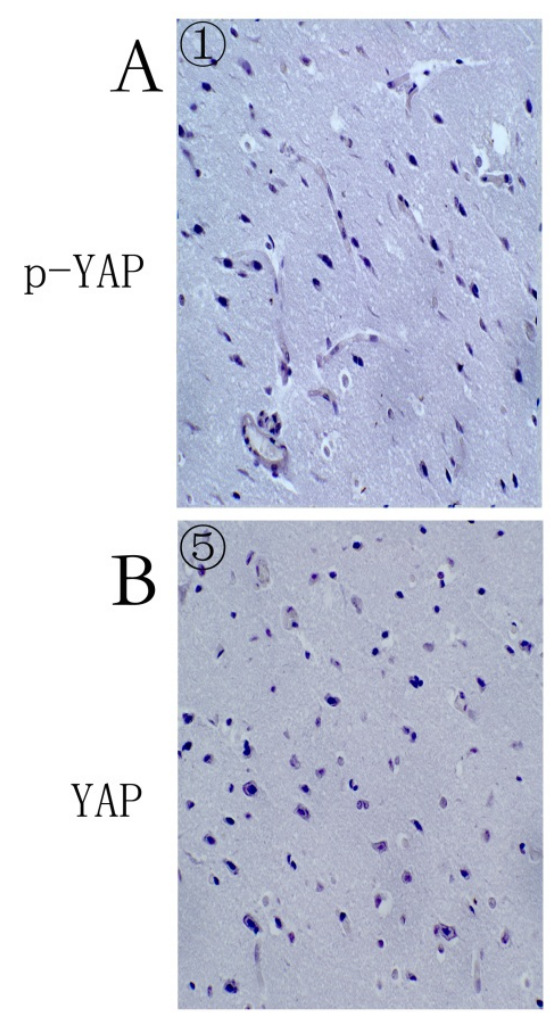

Normal brain
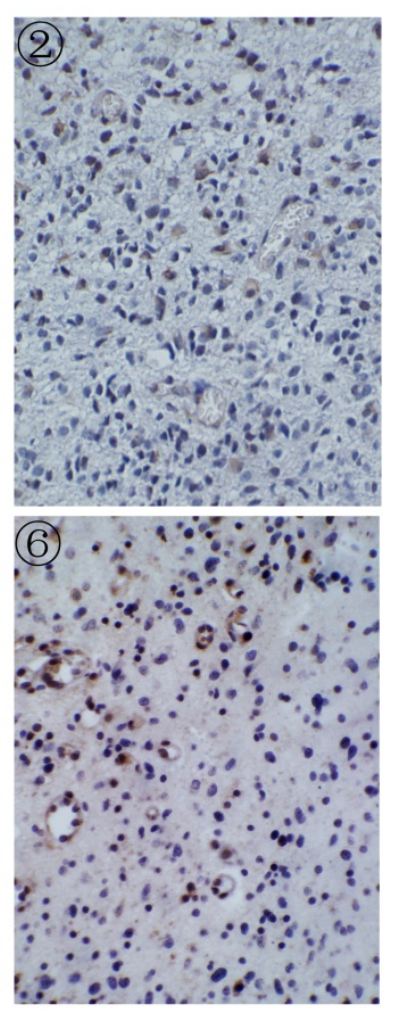

WHO grade II
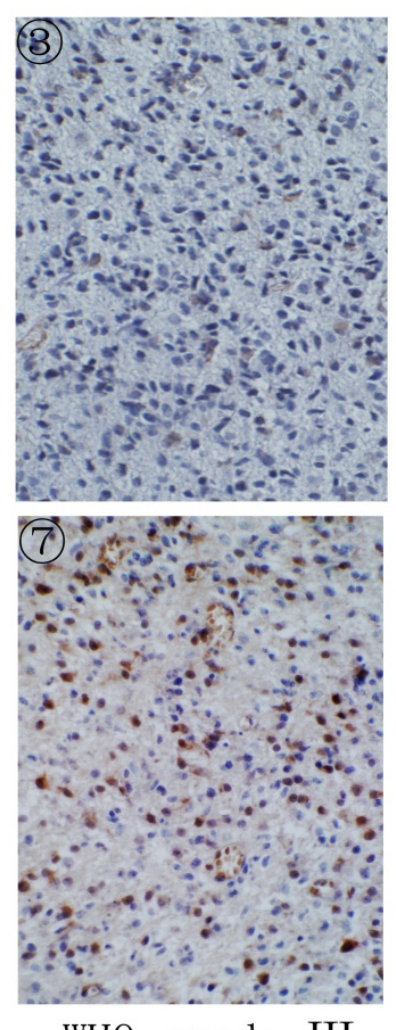

WHO grade III
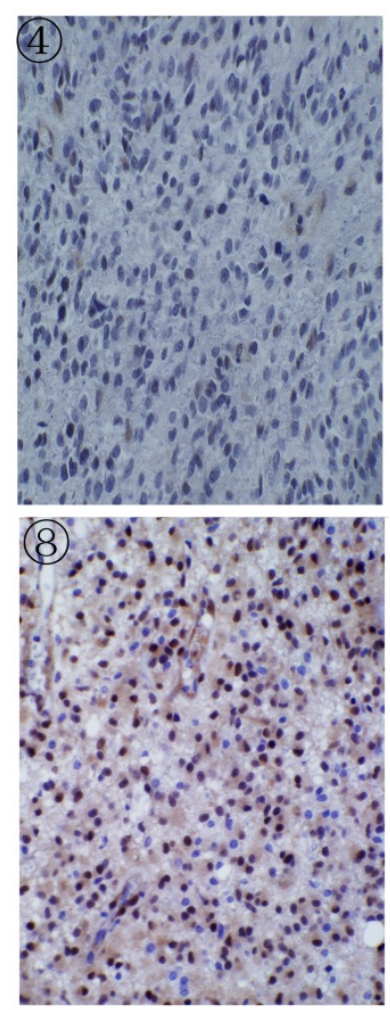

WHO grade IV

Figure 2. Expression of yes-associated protein (YAP) in normal brain tissue and human glioma tissue. (A) $p$-YAP only expressed in cytoplasm of normal brain and glioma tissue. With the enhancement of malignant degree,p-YAP cytoplasm expression decreased.(B) High YAP expressed in cytoplasm and nucleus of glioma tissue. With the enhancement of malignant degree, YAP nucleus expression increased. (8) High YAP expression in glioma tissue with both cytoplasm and nucleus present. Negative YAP nucleus expression in normal brain tissue. Original magnification, $\times 200$ 
Table 3. Primer sequences

\begin{tabular}{l|c} 
Primers & Sequences \\
\hline YAP forward & 5'---AAC TCG GCT TCA GCC ATG A---3' $^{\prime}$ \\
\hline YAP reverse & $5^{\prime}-$--GCT ACG CAG GGC TAA CTC CTG T---3' \\
GAPDH forward & $5^{\prime}-$--GGT GAA GGT CGG AGT CAA CGG---3' \\
\hline GAPDH reverse & $5^{\prime}---$ GAG GTC AAT GAA GGG GTC ATT G---3'
\end{tabular}

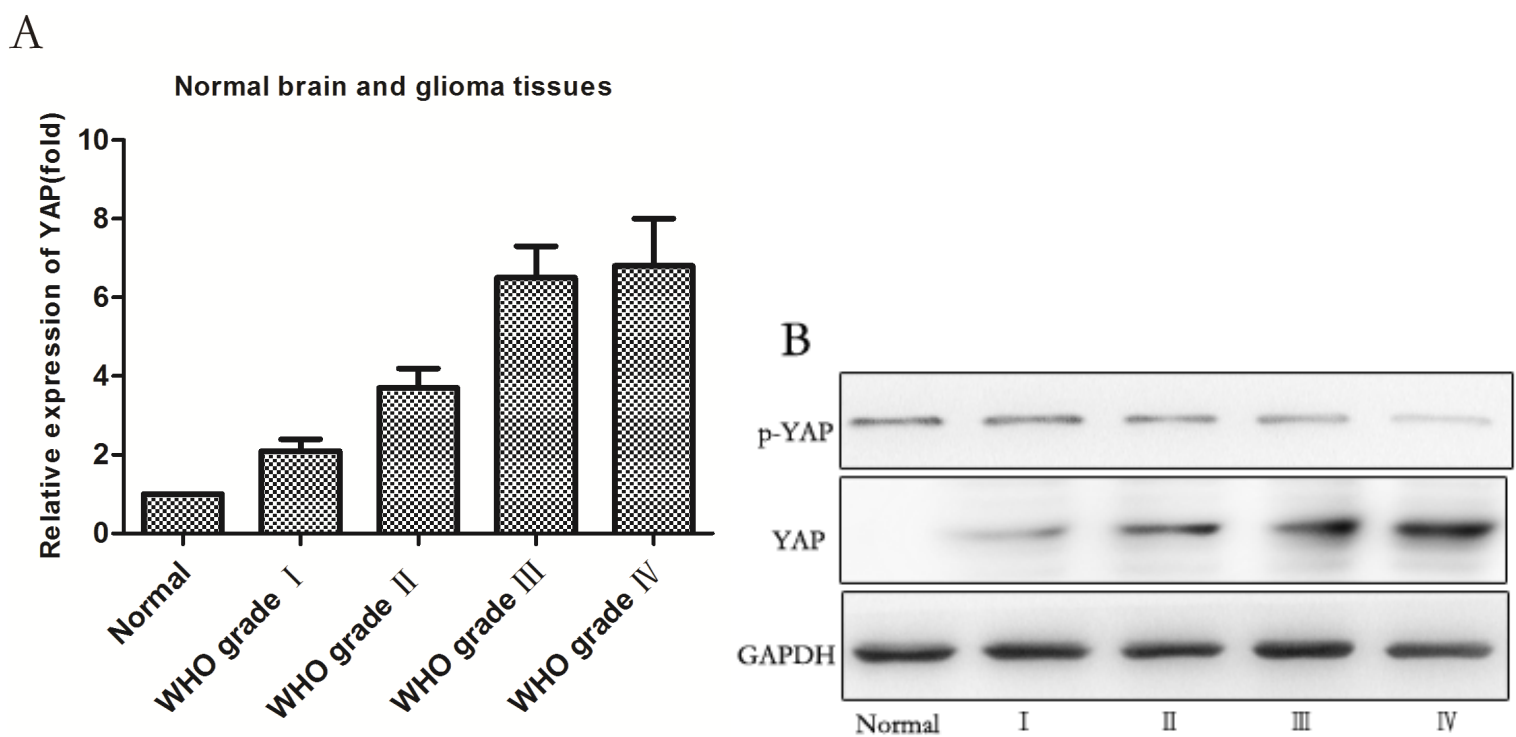

Figure 3.qRT-PCR and western blotting detected the YAP expression. A: qRT-PCR showed the effects of YAP overexpression in glioma tissue compared to the normal brain tissue. B: Control and glioma YAP protein production represent expression in normal brain tissue and glioma tumor tissue by western blotting, respective 

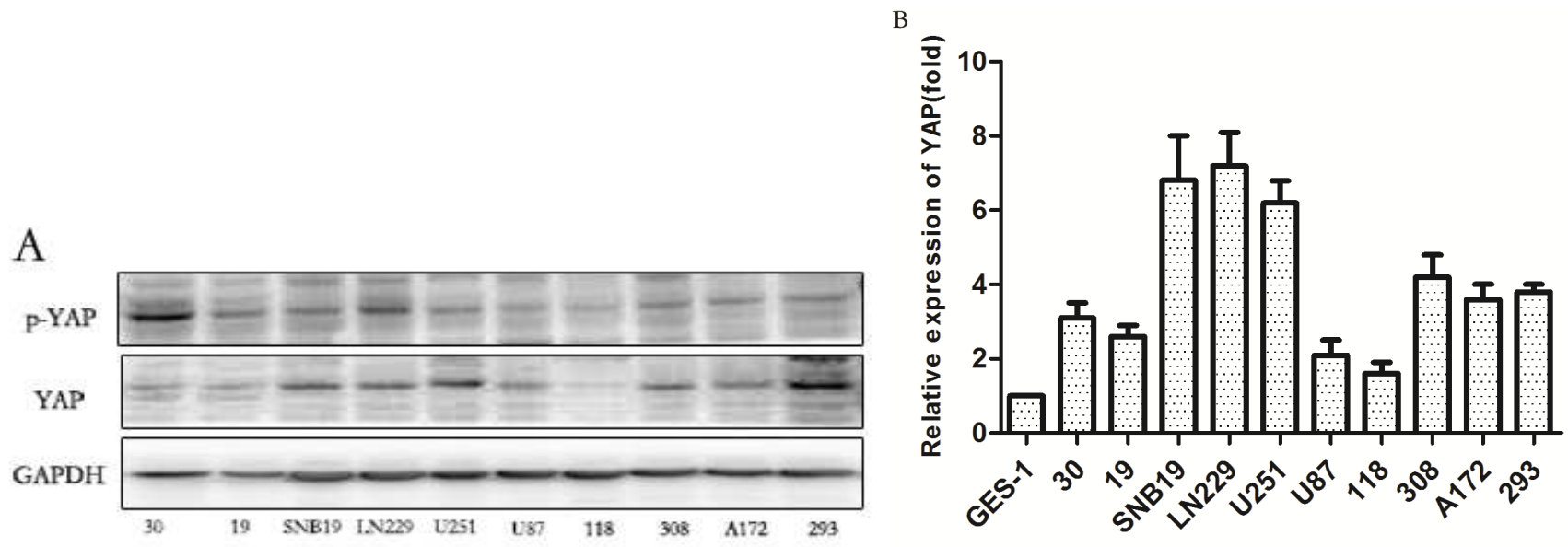

Figure 4. Selection of the specific glioma cell lines.A: Western blot showed that both the level of YAP and ratio of YAP to $p$-YAP varied greatly between cell lines.B:Relative yes-associated protein (YAP) mRNA expression in cell lines. Ten human gioma cell lines and one normal cell line (GES-1)as control, were measured by real-time PCR. The fold change of YAP expression was calculated by the $2^{-\triangle \Delta}$ Ct Method. Columns, mean; bars, SD.
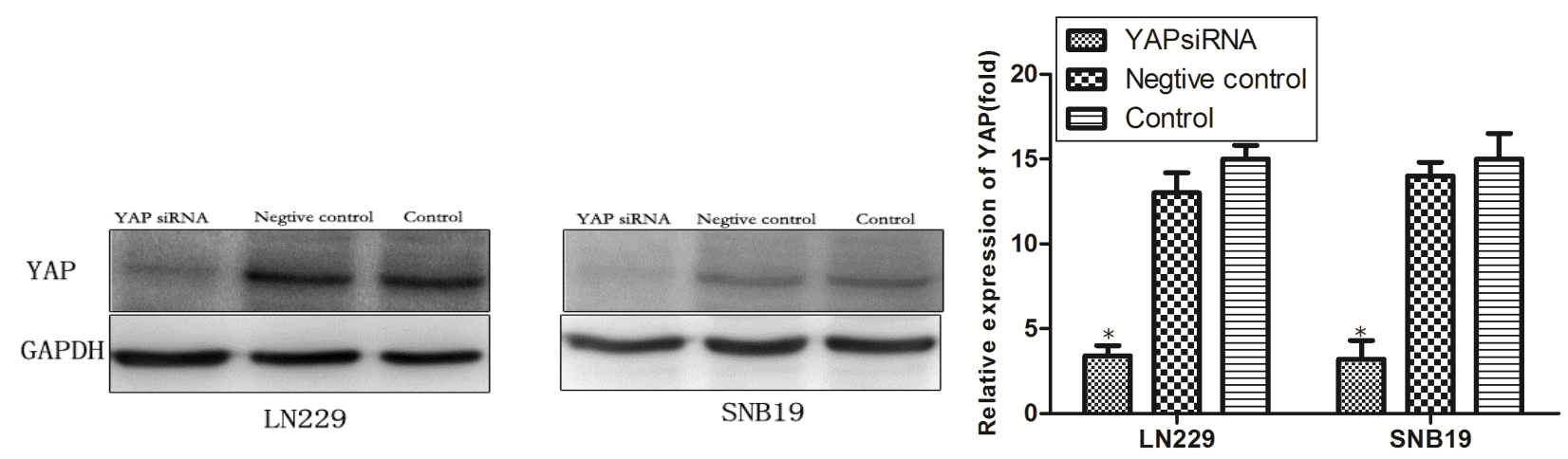

Figure 5.Yes-associated protein (YAP) expression in glioma cells transfected with YAP specific siRNA. The results of western blot and Qrt-PCR proved that YAPsiRNA can decrease the YAP expression significantly. 

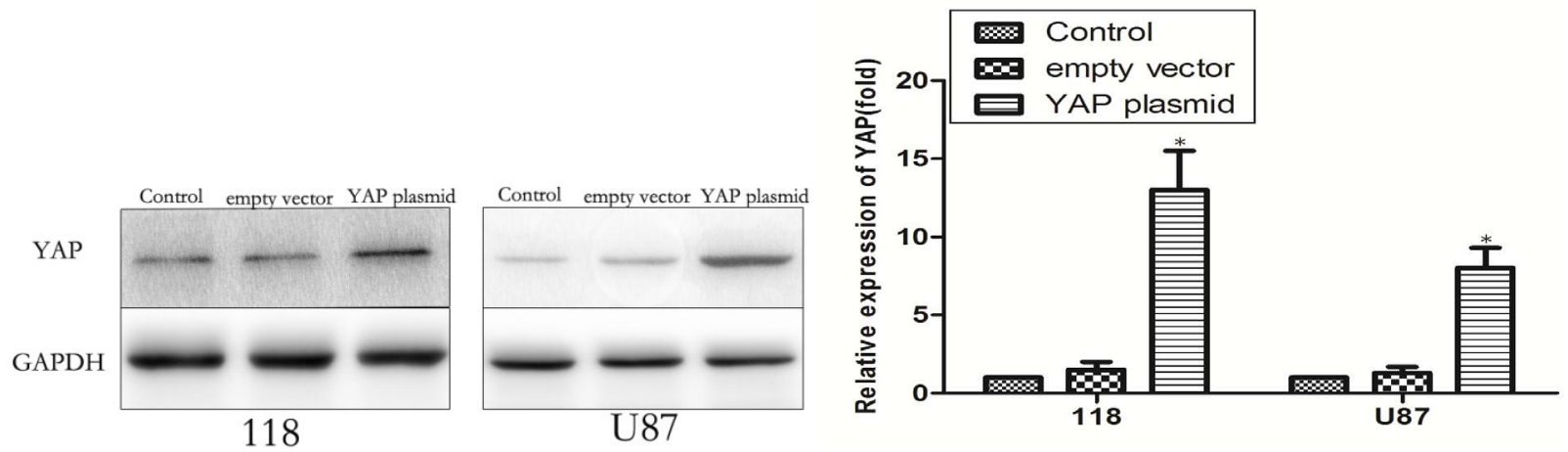

Figure 6. Western blot analysis and qRT-PCR showed the effects of YAP overexpression and YAP silencing of 118 and U87 cells transfected with pcDNA3.1-hYAP and control empty vector.Transfection of pcDNA3.1-hYAP plasmid significantly increased protein levels and YAP mRNA compared with control and empty vector group. 
LN229

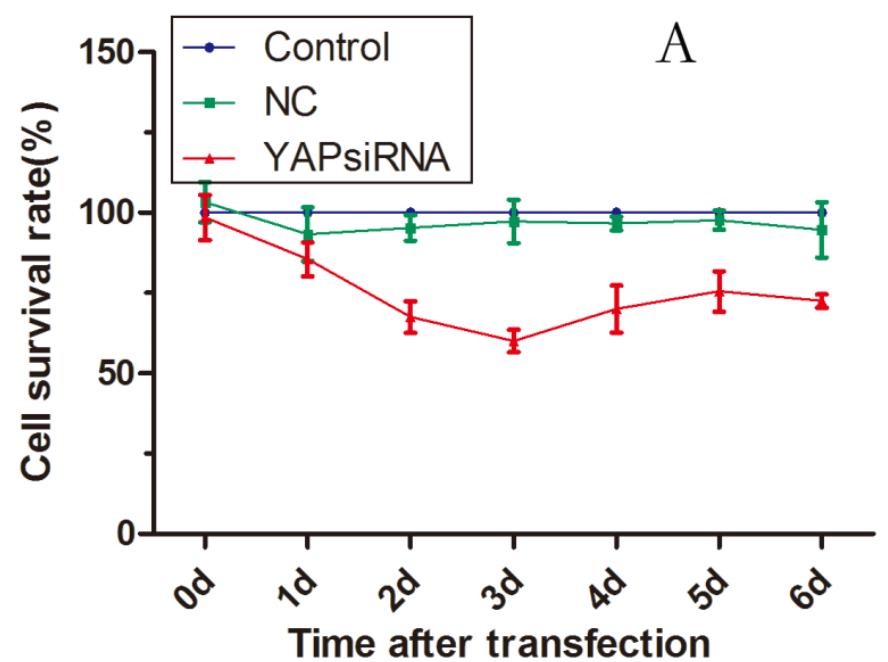

118

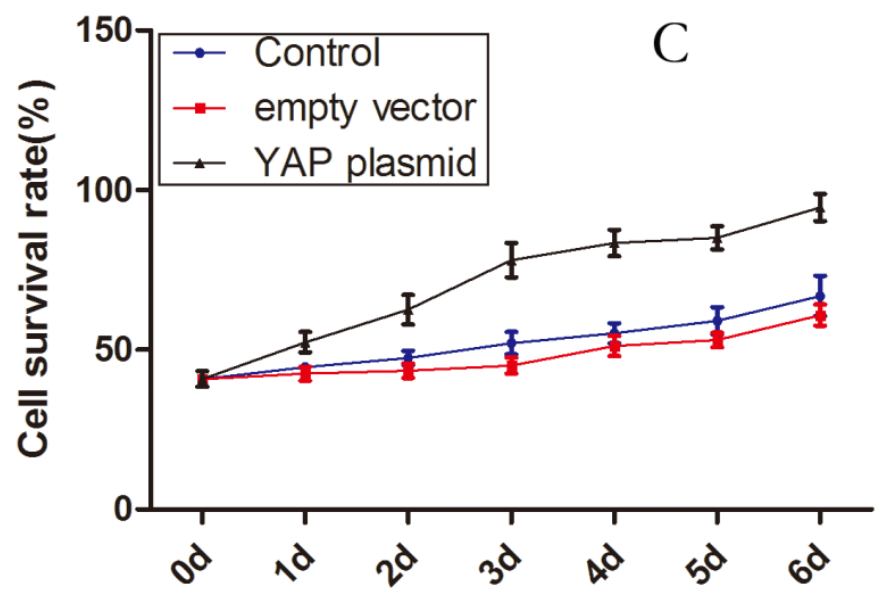

Time after transfection
SNB19

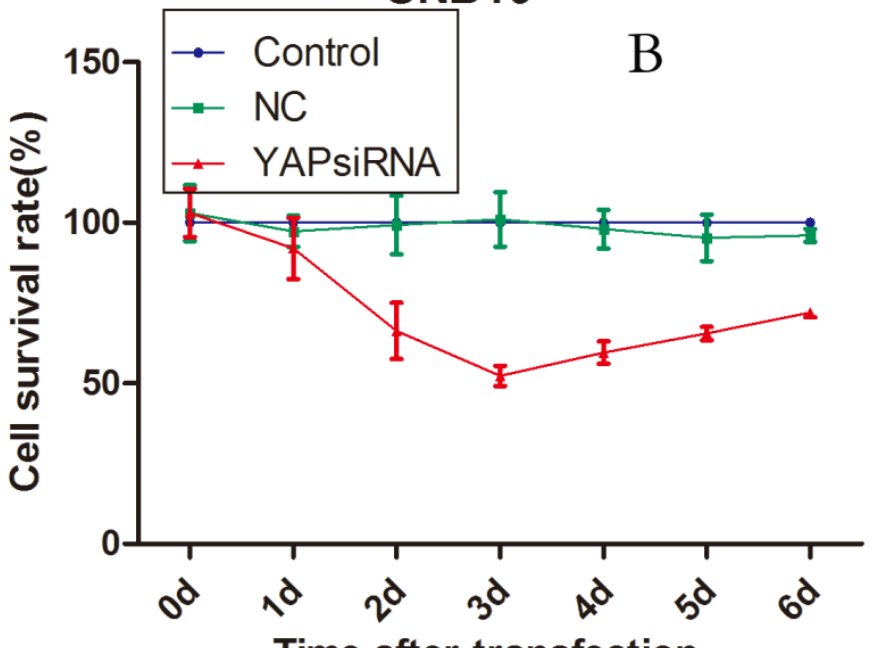

Time after transfection

U87

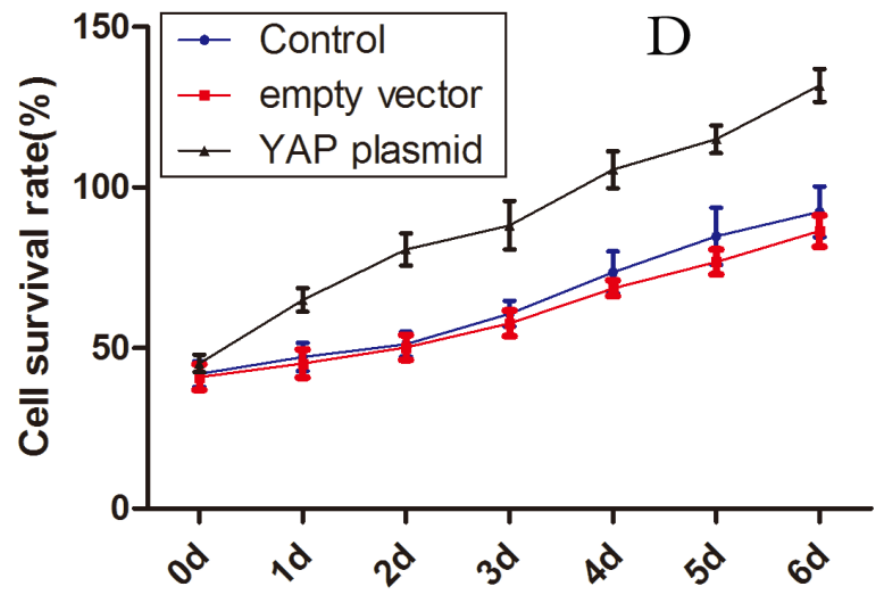

Time after transfection

Figure 7. YAP promotes proliferation of glioma cells.(A.B) YAP depletion by YAPsiRNA in SNB19 and LN229 cells reduced the proliferation rate. (C.D) Overexpression of YAP by YAP plasmid in U87 and 118 cells led to significant increase in the U87 and 118 cell growth rate. 

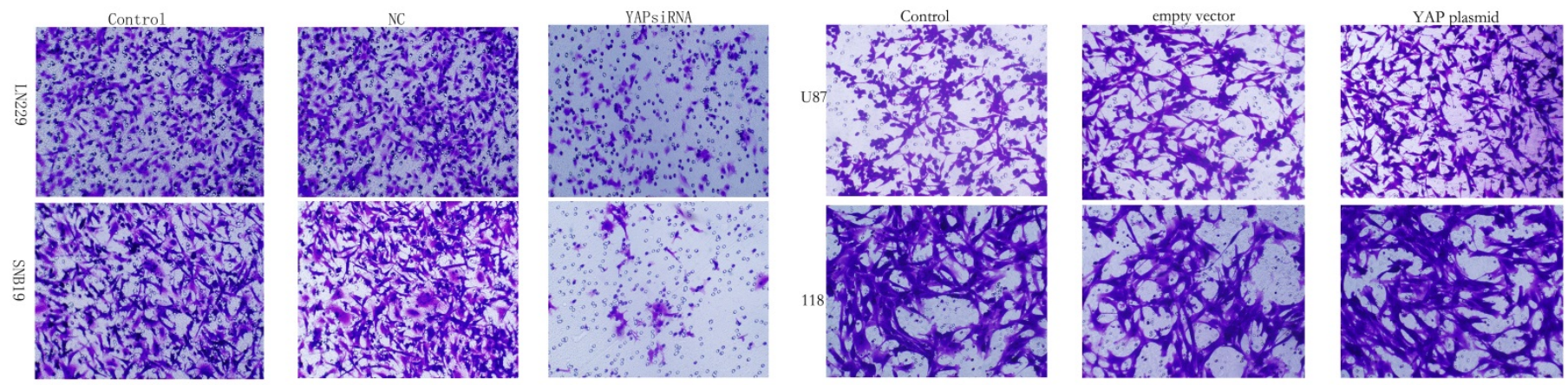

A
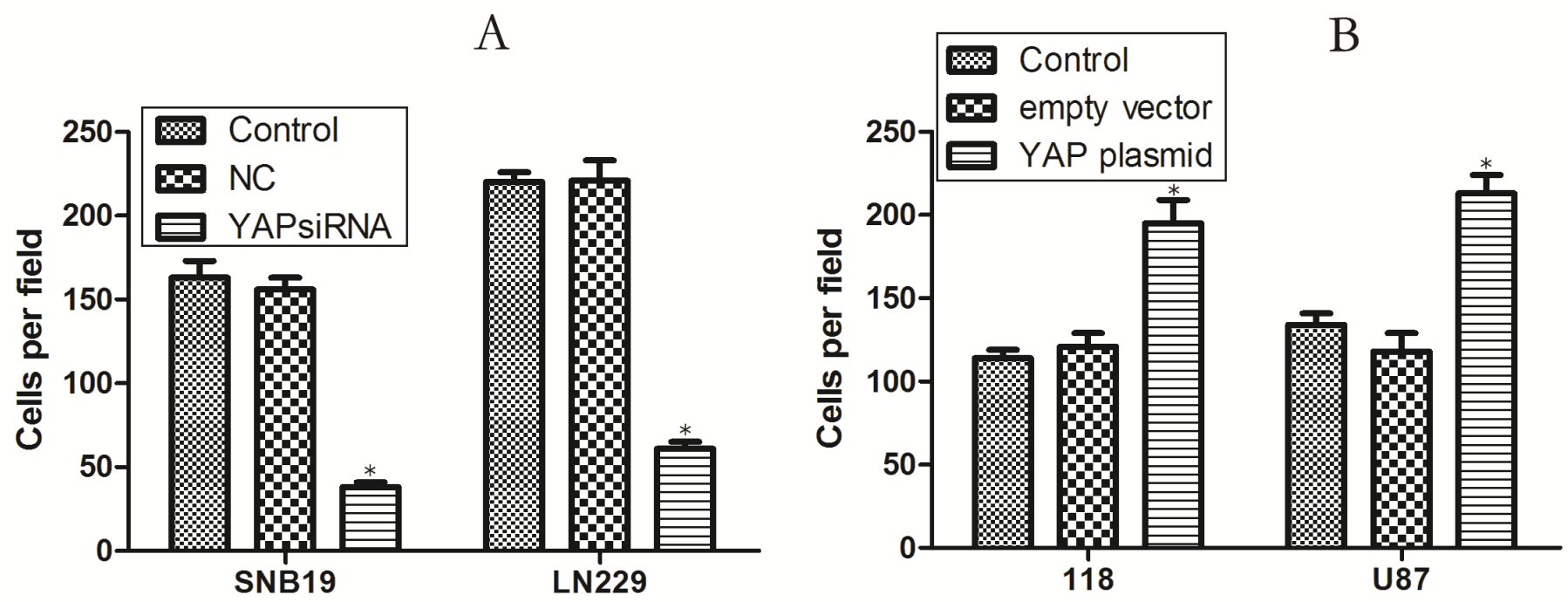

Figure 8. YAP increases the invasion of glioma cells. (A)YAP-depleted SNB19 and LN229 cells showed a weaker invasion $(P<0.05)$ ability compared with control group cells. (B)Overexpression of YAP in U87 and 118 cells stimulated cell invasion $(P<0.05)$ when compared with control and empty vector group. 

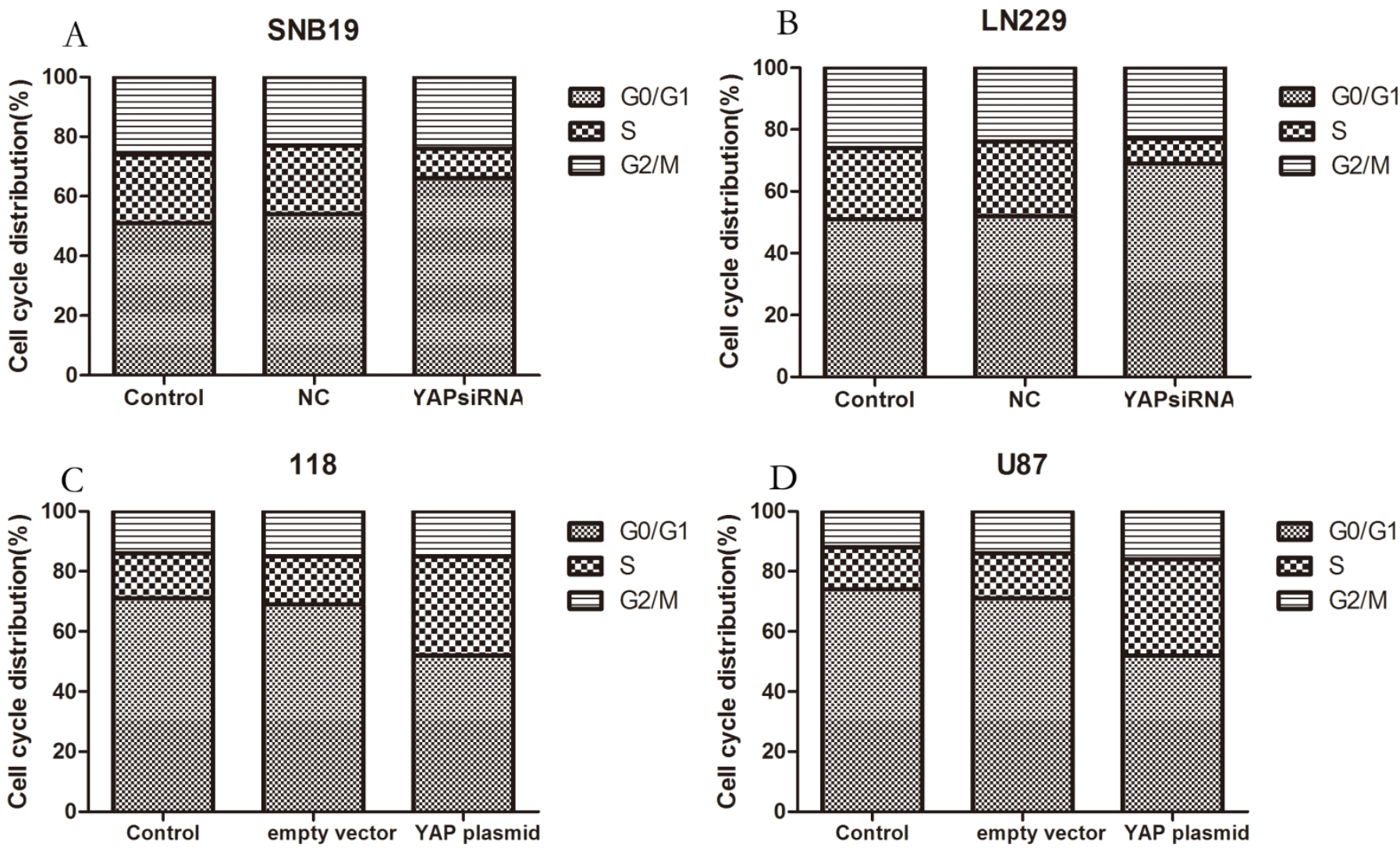

Figure 9. YAP Accelerates the Cycle of glioma cells in the G0/G1 Phase. Treatment with YAPsiRNA resulted in a decrease in the population of cells that were in S phase and a increase of G0/G1 phase. Overexpressed YAP in U87 and 118 cells with the transfection of pcDNA3.1-hYAP plasmid significantly accelerates the cell cycle $(P<0.05)$ when compared with control and empty vector group, indicating that YAP functions as a positive regulator of the cell cycle from G0/G1-to-S transition.
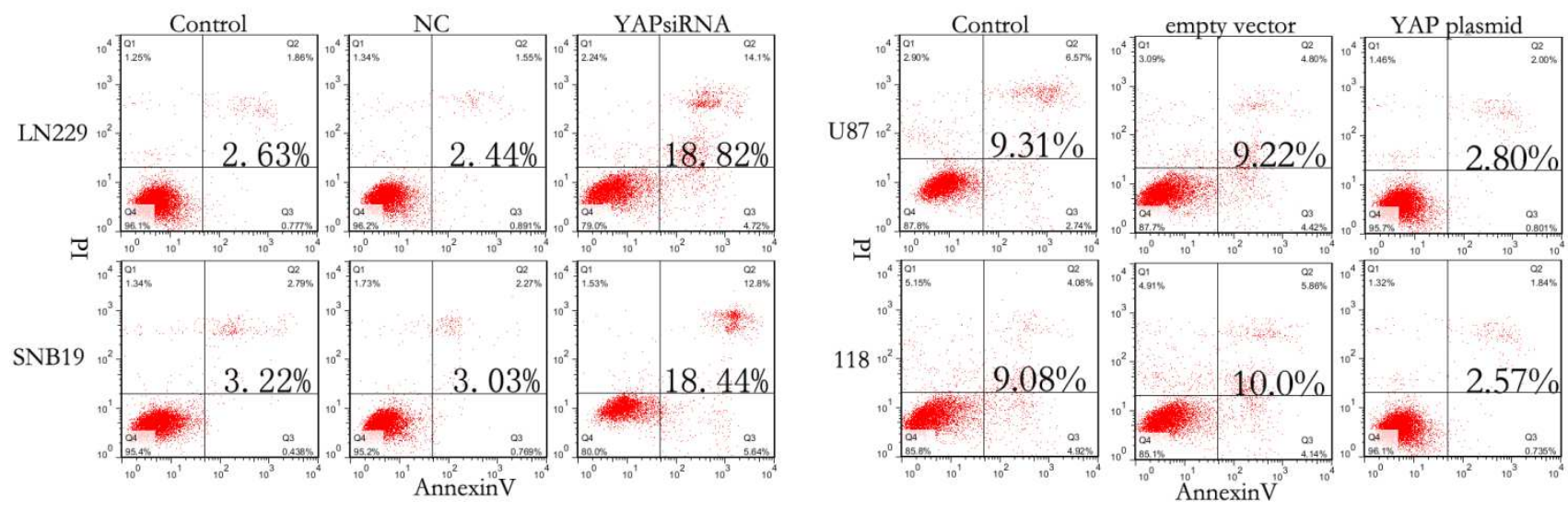

Figure 10. YAP Inhibits Cell Apoptosis. These results indicated that YAP expression played a role in promoting cell proliferation and suppressing cell apoptosis. 

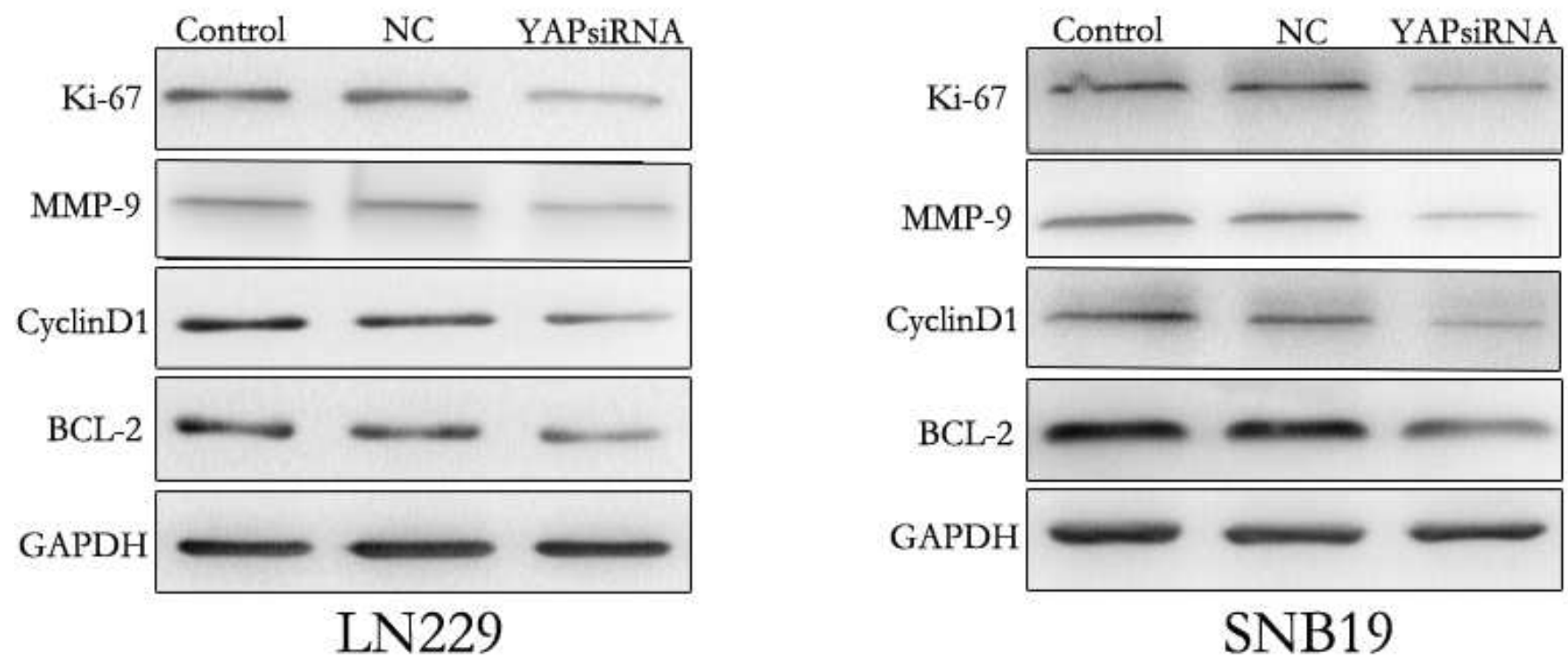

Figure 11:The expression of Ki-67,MMP-9,CyclinD1,BCL-2 detected by Western blotting. The expression of Ki-67,MMP-9,CyclinD1,BCL-2 was downregulated in YAPsiRNA group of LN229 and SNB19 glioma cell lines while up-regulated in YAP plasmid of 118 and U87 glioma cell lines.

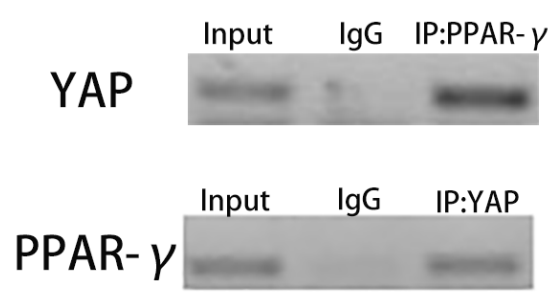

Figure 12. Co-Immunoprecipitation Shows the Combination of YAP and PPARY. Co-Immunoprecipitation experiments were performed on total protein from the LN229 glioma cells showed that YAP interacted with PPARY, suggesting a function at the level of protein. 
118

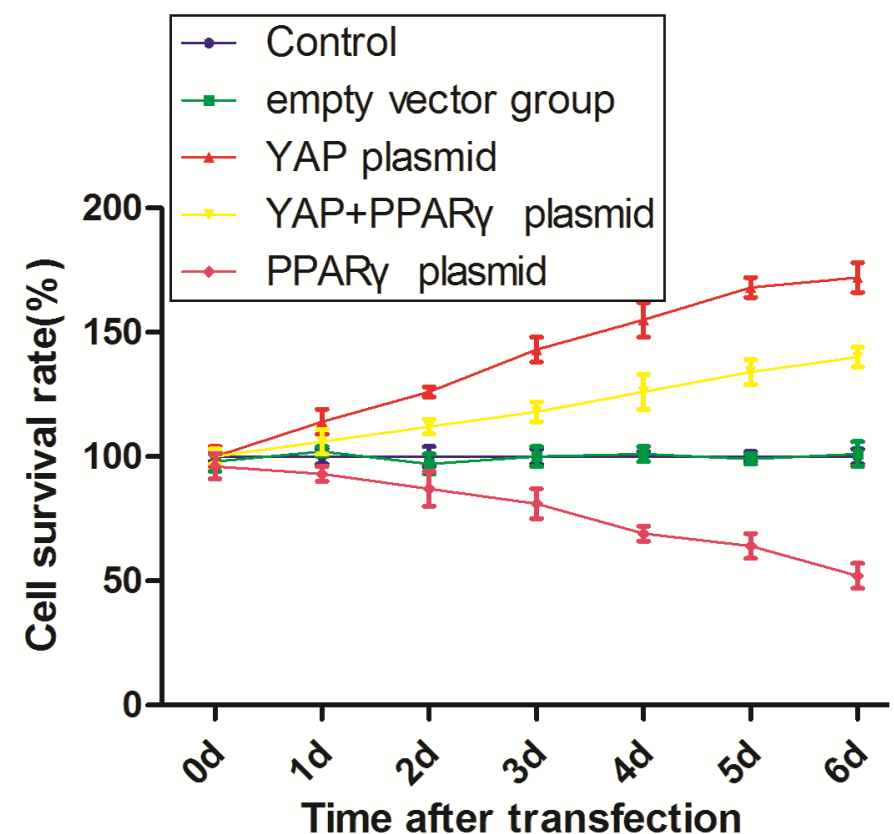

U87

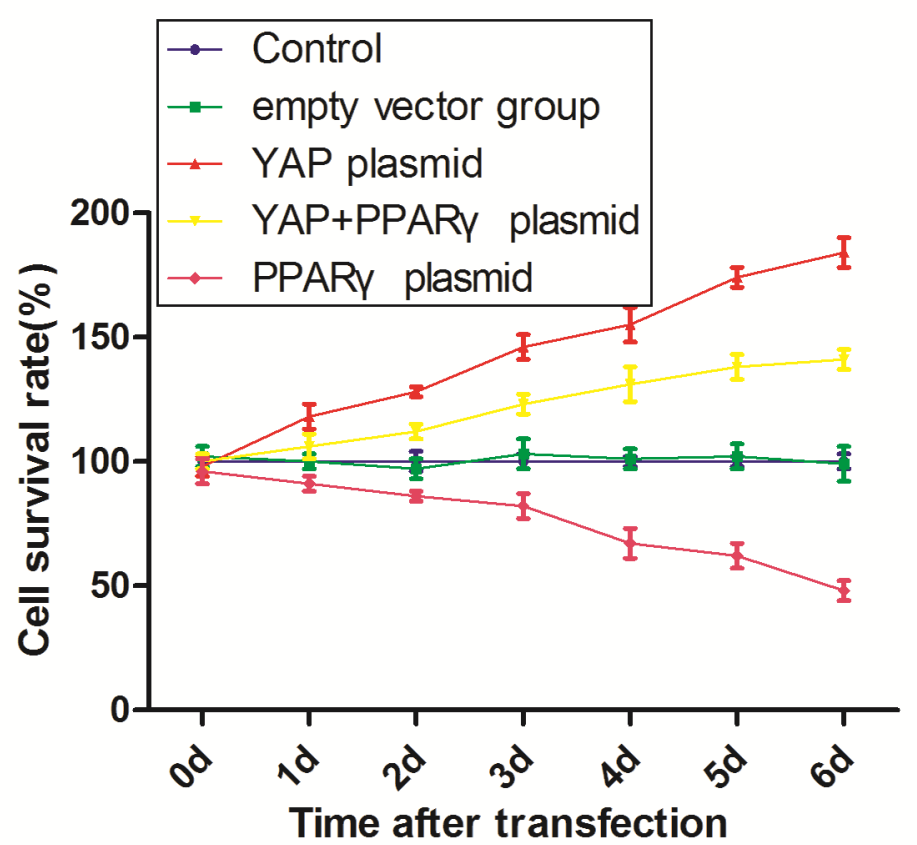

Figure 13. PPARy inhibits proliferation of glioma cells. The figure showed that PPARY inhibited the cell survival rate at a significantly higher rate in 118 and U87 cell. 